\title{
Relationship between individual differences in speech processing and cognitive functions
}

\author{
Jinghua Ou ${ }^{1} \cdot$ Sam-Po Law ${ }^{1} \cdot$ Roxana Fung ${ }^{2}$
}

Published online: 28 April 2015

(C) Psychonomic Society, Inc. 2015

\begin{abstract}
A growing body of research has suggested that cognitive abilities may play a role in individual differences in speech processing. The present study took advantage of a widespread linguistic phenomenon of sound change to systematically assess the relationships between speech processing and various components of attention and working memory in the auditory and visual modalities among typically developed Cantonese-speaking individuals. The individual variations in speech processing are captured in an ongoing sound change-tone merging in Hong Kong Cantonese, in which typically developed native speakers are reported to lose the distinctions between some tonal contrasts in perception and/or production. Three groups of participants were recruited, with a first group of good perception and production, a second group of good perception but poor production, and a third group of good production but poor perception. Our findings revealed that modality-independent abilities of attentional switching/ control and working memory might contribute to individual differences in patterns of speech perception and production as well as discrimination latencies among typically developed speakers. The findings not only have the potential to generalize to speech processing in other languages, but also broaden our understanding of the omnipresent phenomenon of language change in all languages.
\end{abstract}

Sam-Po Law

splaw@hku.hk

1 Division of Speech and Hearing Science, the University of Hong Kong, Pokfulam, Hong Kong SAR

2 Department of Chinese and Bilingual Studies, the Hong Kong Polytechnic University, Hung Hom, Hong Kong SAR
Keywords Individual differences $\cdot$ Speech perception · Speech production $\cdot$ Attentional switching $\cdot$ Working memory $\cdot$ Inhibitory control

Speech processing is fundamental to any verbal communication. The ease with which speech is perceived underscores the complexity of the underlying perceptual and cognitive mechanisms, which are capable of rapidly decoding complex acoustic signals and extracting the intended messages (Holt \& Lotto, 2010). The ability to selectively attend to relevant acoustic signal in the speech while inhibiting irrelevant information is proposed to be essential in fine-tuning speech perception during development (Conboy, Sommerville, \& Kuhl, 2008; Jusczyk, 2002). The relevance of cognitive abilities to individual differences in speech processing is evidenced by studies in populations with developmental disorders, such as autism spectrum disorder (see Haesen, Boets, \& Wagemans, 2011, for a review), or developmental dyslexia (e.g., Facoetti et al., 2010; Ruffino et al., 2010). Particularly, deficits in phonological processing among individuals with developmental dyslexia have been attributed to difficulties in directing and switching auditory attention to rapidly presented acoustic signals (Hari \& Renvall, 2001; Lallier et al., 2010). Thus, the flexibility of the cognitive system pertaining to attention is likely to be associated with individual differences in speech processing.

Apart from language disorders, much of the research in speech processing of typically developed speakers has characterized individual differences by employing participants differing in learning/training experience, including second language (L2) learning (Díaz, Baus, Escera, Costa, \& SebastiánGallés, 2008; Lengeris \& Hazan, 2010) and musical training (George \& Coch, 2011; Parbery-Clark, Skoe, Lam, \& Kraus, 2009) to assess its relationship with cognitive abilities. For 
instance, Parbery-Clark et al. (2009) found that the ability to perceive speech in background noise was positively correlated with auditory attention and working memory among individuals differing in musical training. The association of individual differences in speech perception and auditory attentional switching is also shown among L2 learners (Díaz et al., 2008). However, few investigations of individual differences in speech processing have been conducted with native speech perception among typically developed speakers. This situation is perhaps due to an implicit assumption that native speakers are comparably skilled in processing all speech sounds of their language. This "uniformity assumption" (Andrews, 2012) has been questioned in studies of word recognition and sentence reading, where significant individual differences are also found among adult skilled readers (Veldre \& Andrews, 2014). A recent study by Lev-Ari and Peperkamp (2014) indeed examined individual differences in native speech perception and reported that inhibitory skills influenced how native listeners might process synthesized phonetic cues during an auditory lexical decision task, which led to individual variations in phonological representations in perception and production.

In the present study, we took advantage of a unique opportunity in sound change to investigate individual differences in native speech processing among typically developed speakers. It is well established in sociolinguistic studies that the phonological system of any language is constantly undergoing some form of change (Labov, 1994, and references therein). This usually protracted phenomenon is realized in the form of variable patterns of perception and production across individuals in a community that is assumed to be otherwise linguistically homogeneous (Baker, Archangeli, \& Mielke, 2011; Beddor, 2009; Koenig, Lucero, \& Perlman, 2008). Therefore, the present investigation aims to understand individual variations in speech processing, particularly the mismatch between perception and production emerging from an ongoing sound change with respect to internal factors of cognitive abilities. The specific context of sound change under investigation is tone merging in Hong Kong Cantonese (HKC).

Tonal languages, such as Chinese, comprise $70 \%$ of the world's languages (Yip, 2002). Among all Chinese dialects, the tone inventory of HKC is considered one of the richest. There are six contrastive tones for open syllables in HKC, namely T1 (high-level tone), T2 (high-rising tone), T3 (midlevel tone), T4 (low-falling/extra-low-level tone), T5 (lowrising tone) and T6 (low-level tone). The pitch contours of these six tones are shown in Fig. 1. Similar to segmental sounds in many languages of the world (Labov, 1994), this highly complex tone system is in the process of merging, in which particular pairs of tones become less distinctive in perception and/or production. Observations by phonologists and sociolinguists have revealed that three tone contrasts are

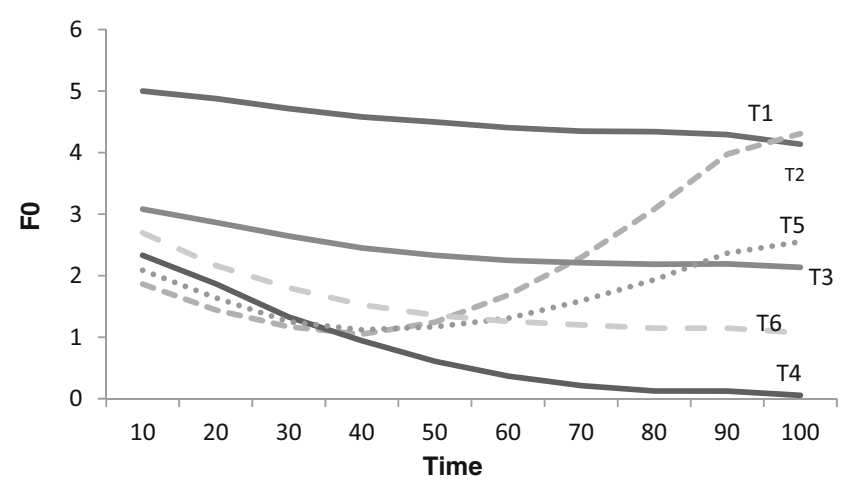

Fig. 1 Normalized fundamental frequency (F0) patterns of the six contrastive tones in Hong Kong Cantonese

collapsing: the low-rising and high-rising tones (T2/T5), the mid-level and low-level tones (T3/T6), and low-falling and low-level tones (T4/T6; Bauer, Cheung, \& Cheung, 2003; Law, Fung, \& Kung, 2013; Mok, Zuo, \& Wong, 2013). Specifically, a significant proportion of Cantonese adult speakers can no longer distinguish the two rising tones $\mathrm{T} 2 /$ $\mathrm{T} 5$ in perception and/or production. While most speakers can discriminate the mid-level and low-level tones T3/T6 in perception, this contrast is not maintained in production. The opposite pattern has been observed for low-falling and lowlevel tones $\mathrm{T} 4 / \mathrm{T} 6$, where the distinction in production is preserved whereas perception is not.

A few studies have indeed explored the relationship between cognitive functions and variability in speech processing in the context of sound change. Yu (2010) found that English-speaking individuals who rated themselves as having low communication skills and attention switching abilities on Autism Spectrum Quotient subscales were less likely to take into account context-induced effects, such as perception of sibilants /s/ or / / when followed by rounded vowels /u/ to recover the intended message. For these speakers, finegrained phonetic distinctions tended to be maintained in perception. Although the study seemed to suggest a link between the distinctiveness of speech perception and the ability of attentional switching, the latter was based on subjective rating. It would be more desirable to use objective tools to assess an individual's cognitive functions. Law et al. (2013) examined brain responses to T4 and T6 in Cantonese of two groups of typically developed adult speakers using eventrelated potential (ERP). Behaviorally, both groups of participants could produce all six tones distinctively; they differed only in their perception of the T4/T6 contrast. As expected, the two participant groups showed differential responses to the T4/T6 contrast in mismatch negativity (MMN), which reflects sensitivity to sound distinctions (Näätänen, Paavilainen, Rinne, \& Alho, 2007). Interestingly, the authors also noticed that the participants who failed to discriminate T4 and T6 perceptually exhibited a tendency of weaker P3a than the controls in all experimental conditions, suggesting a possible 
difference in attention switching, auditory attention, and/or auditory memory (Polich, 2003). However, the observations in Law et al. (2013) remain impressionistic; further evidence for the relationship between variability in speech perception and cognitive abilities is necessary.

In the present investigation, three groups of typically developed native speakers of HKC with comparable language and musical backgrounds were recruited. The participant groups represented different patterns of performance on tone perception and production, that is, good perception $[+\mathrm{Per}]$ and good production [+Pro], good perception [+Per] but poor production [-Pro] of the T2/T5 distinction, and good production [+Pro] but poor perception [-Per] of the T4/T6 contrast. In light of previous findings showing relationships between individual differences in speech perception and cognitive abilities, including working memory, attention switching, and inhibitory control, a battery of cognitive tasks assessing various components of attention and working memory in the auditory and visual modalities was administered to all participants. Only published tools and tasks were employed for ease of comparison with results of previous studies. The findings will provide insights not only to the study of sound change but, more importantly, also to our understanding of speech processing among typically developed individuals.

\section{Method}

\section{Ethics statement}

All participants gave informed consent in compliance with an experimental protocol approved by the University of Hong Kong Research Ethics Committee for Non-Clinical Faculties, and were paid for their participation in the study.

\section{Participants}

A total of 138 native speakers of Cantonese, all born and raised in Hong Kong, were recruited. No speaker reported a history of hearing abnormalities. They first participated in a tone perception and a tone production task (see details below). The performance on these tasks revealed whether a participant could distinguish all tones in perception and production or failed to do so for a specific tone contrast in one or both modalities. In the end, 60 participants were invited back to carry out a series of cognitive tasks.

The 60 participants were classified into three groups. Table 1 presents the characteristics of the three participant groups in terms of their performance on tone perception, tone production, and musical background. One group could distinctively perceive and produce all six Cantonese tones ([+ Per+Pro], $N=20$, female $=7$ ); a second group could perceive all tones but fail to produce T2 and T5 distinctively ([+Per-
Pro], $N=20$, female $=11$ ), and the third group could produce all tones distinctively but fail to perceive the difference between T4 and T6 ([-Per+Pro], $N=20$, female = 12). As shown in Table 1 , the mean accuracy of $\mathrm{T} 2$ and $\mathrm{T} 5$ production was significantly lower for the [+Per-Pro] than [+Per+Pro] and [Per+Pro] (post hoc Bonferroni correction $p<.001$ for both), and the mean score of T4 versus T6 perception was significantly lower for [-Per+Pro] than the other two groups (both $p$ s $<.001)$. The three groups were matched in age, $F(2,57)=$ $2.45, p=.095$, years of formal education, $F(2,57)=.39, p>$ .250 , and musical background in onset and duration of training, onset: $F(2,57)=.24, p>.250$; duration: $F(2,57)=.16, p$ $>.250$.

\section{Tasks and procedures}

\section{Tone perception and production tasks}

\section{Stimuli}

To control for syllable effect, only one CV root [fu] was used to derive the six tones. The six syllables were produced and recorded by a native female Cantonese speaker and served as the stimuli of the perception task. The length of the stimuli was $500 \mathrm{~ms}$ and the intensity $70 \mathrm{~dB}$ SPL. For the production task, the six syllables were represented by six Chinese characters, that is, 夫 fu1 “husband,”苦 fu2 “bitter,” 褲 fu3 “trousers,”符 fu4 “symbol,”婦 fu5 “women,” and 負 fu6 "negative."

\section{Procedures}

The production task was carried out before the perception task to eliminate any priming effect.

The perception task was an AX discrimination test. Thirtysix tone pairs (6 AA pairs and $30 \mathrm{AB}$ pairs counterbalanced in order of syllables) were repeated 10 times each, giving a total of 360 stimuli. The participants had to indicate as soon as possible whether the tones presented were the same or different. Both accuracy and reaction time were collected.

In the production task, the six syllables were embedded in different positions of two sentence carriers: $/ \mathrm{y}^{13} \mathrm{ji}^{21 / 55} \mathrm{ka}^{55}$ $\mathrm{t} \mathrm{k}^{2} \mathrm{tsi}^{22} /$ "I am now reading the character" and / nei ${ }^{55} \mathrm{k}^{33}$ $\mathrm{tsi}^{22} \mathrm{~h} \mathrm{i}^{22}$ / "This character is _." The 12 stimuli (6 syllables x 2 carriers) were repeated 10 times each, thus generating 120 trials. The participants were instructed to read aloud the sentence at a normal speech rate. The speech outputs were recorded digitally for phonetic transcription by a native Cantonese speaker with training in phonetics who was blind to the target stimuli, and $10 \%$ of the trials were randomly selected and transcribed by a second native Cantonese speaker, also blind 
Table 1 Musical background of participants in different groups and their accuracy of tone perception and production

\begin{tabular}{|c|c|c|c|c|c|c|}
\hline & \multicolumn{2}{|c|}{$[+$ Per+Pro $]$} & \multicolumn{2}{|c|}{$[+$ Per-Pro $]$} & \multicolumn{2}{|c|}{$[-$ Per+Pro $]$} \\
\hline & \multicolumn{2}{|c|}{$(N=20)$} & \multicolumn{2}{|c|}{$(N=20)$} & \multicolumn{2}{|c|}{$(N=20)$} \\
\hline & $M$ & $S D$ & $M$ & $S D$ & $M$ & $S D$ \\
\hline Age & 22.60 & 2.42 & 20.55 & 2.54 & 22.85 & 5.16 \\
\hline Years of education & 17.1 & 1.8 & 16.6 & 2.6 & 17.2 & 2.8 \\
\hline \multicolumn{7}{|l|}{ Tone perception } \\
\hline Distinguishing T4/T6 & 0.97 & 0.03 & 0.92 & .008 & $0.70^{\mathrm{a}}$ & 0.11 \\
\hline Distinguishing all other tones & 0.99 & 0.02 & 0.98 & 0.01 & 0.98 & 0.01 \\
\hline \multicolumn{7}{|l|}{ Tone production } \\
\hline Distinguishing T2-T5 & 0.99 & 0.02 & $0.63^{\mathrm{b}}$ & 0.15 & 0.93 & 0.11 \\
\hline All other tones & 0.98 & 0.05 & 0.89 & 0.95 & 0.95 & 0.05 \\
\hline \multicolumn{7}{|l|}{ Musical background } \\
\hline Onset & 5.85 & 6.79 & 4.95 & 4.70 & 6.25 & 6.58 \\
\hline Duration & 3.45 & 4.43 & 3.90 & 4.41 & 4.25 & 4.47 \\
\hline
\end{tabular}

Note. ${ }^{a}$ The accuracy of distinguishing T4/T6 in perception was significantly lower for [-Per+Pro] than the other two groups (post hoc Bonferroni correction $p<.001$ for both).

$\mathrm{b}$ The accuracy of distinguishing T2 and T5 in production was significantly lower for [+Per-Pro] than the other two groups (both $p \mathrm{~s}<.001$ )

to the targets. The interrater reliability reached a $95 \%$ agreement.

\section{Cognitive measures of attention and working memory}

Auditory and visual attention were assessed using the six subtests in the Test of Everyday Attention (TEA; Robertson, Ward, Ridgeway, \& Nimmo-Smith, 1994), the Attentional Network task (ANT; Fan, McCandliss, Sommer, Raz, \& Posner, 2002), and the test of auditory attentional shifting-auditory stream segregation (Lallier et al., 2010). Auditory and visual working memory were, respectively, evaluated by digit span backward and the subscales of visual processing speed-symbol search, coding and cancellation in the Wechsler Adult Intelligence Scale, Fourth Edition (WAIS-IV; Wechsler, 2010), respectively. The subtests of the TEA and WAIS-IV were administered following the respective instruction manuals. All test instructions were given in Cantonese, which were initially translated from the English manuals. The Cantonese instructions underwent a back translation into English to ensure the accuracy and completeness of the translation. All the measures were scaled-scored accordingly.

\section{Data analyses}

A series of one-way ANOVAs was performed on the overall RTs of tone perception, and the scores of all the measures of attention and working memory to detect any difference among groups. The RTs of tone perception with incorrect responses and outlier responses greater than \pm 3 standard deviations of the group mean were removed. In addition, as the participants in the [-Per+Pro] group failed to reliably perceive the distinction between $\mathrm{T} 4$ and T6, RTs to these two tones were not valid observations of discrimination. Thus, trials involving T4 and T6 were excluded in the calculation of the individual overall RT for all participants. Post hoc comparisons were conducted if a significant group effect was found.

The relationships between discrimination latencies and cognitive abilities were then explored in two steps. First, in order to reduce the number of predictor variables, three composite scores were computed by summing the $z$ scores of the TEA visual subtests (TEA visual), auditory subtests (TEA auditory), and the three subtests in WAIS-IV (visual WM), respectively. Pearson correlations among the overall RT and the six scores-TEA visual, TEA auditory, executive control reflected by the ANT, auditory stream segregation, digit span backward, and visual WM-were considered prior to regression analyses. Second, stepwise multiple regression analyses were conducted with the overall RT as the predicted variable and cognitive measures that were significantly correlated with the overall RTs as the predictor variables.

\section{Results}

The performance on tone perception in RT and cognitive measures of different participant groups is shown in Table 2. An omnibus ANOVA revealed that the overall RTs of tone perception significantly differed across groups, $F(2,57)=7.87$, $p=.001, \eta^{2}=.22$. Post hoc test using the Bonferroni correction showed that discrimination latencies were significantly shorter in $[+$ Per+Pro] than that of $[+$ Per-Pro $](p=.004)$ and 
Table 2 Performance on tone perception in RT and cognitive measures of different participant groups

\begin{tabular}{|c|c|c|c|c|c|c|c|}
\hline \multirow[t]{2}{*}{ Measures } & \multicolumn{2}{|c|}{$[+$ Per+Pro $]$} & \multicolumn{2}{|c|}{ [+Per-Pro] } & \multicolumn{2}{|l|}{ [-Per+Pro] } & \multirow[t]{2}{*}{$p$} \\
\hline & $M$ & $S D$ & $M$ & $S D$ & $M$ & $S D$ & \\
\hline Overall RT (ms) in tone perception & $1,000.38$ & 86.76 & $1,165.95$ & 205.78 & $1,127.89$ & 179.49 & $.001 *$ \\
\hline \multicolumn{8}{|l|}{ Test of Everyday Attention } \\
\hline Map Search $1^{\mathrm{a}}(\mathrm{MS} 1)$ & 11.45 & 2.16 & 11.50 & 2.26 & 11.00 & 2.47 & .752 \\
\hline Map Search $2^{\mathrm{b}}(\mathrm{MS} 2)$ & 9.05 & 2.14 & 9.15 & 2.28 & 8.90 & 3.19 & .954 \\
\hline Visual Elevator $1^{\mathrm{c}}$ (VE1) & 11.10 & 2.67 & 11.05 & 2.37 & 11.80 & 2.31 & .562 \\
\hline Visual Elevator $2^{\mathrm{d}}$ (VE2) & 14.55 & 3.47 & 13.45 & 2.82 & 12.10 & 1.94 & $.002 *$ \\
\hline Telephone Search (TS) & 15.85 & 3.50 & 15.75 & 3.80 & 13.10 & 2.83 & .020 \\
\hline Elevator Counting with Distractor (ECD) & 12.40 & 1.50 & 11.65 & 2.01 & 11.85 & 2.50 & .453 \\
\hline Elevator Counting with Reversal (ECR) & 11.95 & 1.96 & 11.75 & 1.92 & 11.50 & 1.88 & .019 \\
\hline Telephone Search while Counting (TSC) & 13.25 & 3.29 & 11.00 & 3.18 & 11.00 & 2.75 & .035 \\
\hline ANT-executive control & 70.55 & 18.82 & 68.22 & 21.28 & 74.59 & 27.71 & .675 \\
\hline Auditory stream segregation (AS) & 61.67 & 34.90 & 72.32 & 37.74 & 67.42 & 44.96 & .680 \\
\hline Digit span backward (DSB) & 7.95 & 1.57 & 7.65 & 1.57 & 7.75 & 1.68 & .775 \\
\hline \multicolumn{8}{|l|}{ WAIS-IV } \\
\hline Symbol Search (SS) & 15.35 & 2.82 & 14.95 & 3.21 & 14.55 & 3.03 & .357 \\
\hline Coding (CD) & 16.65 & 2.77 & 15.70 & 3.29 & 15.60 & 2.99 & .446 \\
\hline Cancellation (CA) & 13.00 & 2.20 & 12.15 & 2.98 & 11.95 & 2.625 & .492 \\
\hline
\end{tabular}

Note. ${ }^{\mathrm{a}}$ Correct responses in 1 minute. ${ }^{\mathrm{b}}$ Correct responses in 2 minutes. ${ }^{\mathrm{c}}$ Number of correct responses in the VE task. ${ }^{\mathrm{d}}$ Time-per-switch in the VE task (switch refers to the elevator switching in direction, i.e., from going up to going down and vice versa). Adjusted significance threshold equals $p=.05 / 15$ $=0.003$

$* p<.003$

[-Per+Pro] $(p=.003)$, whereas no significant difference was observed between the [+Per-Pro] and [-Per+Pro] groups (see Fig. 2). In terms of the cognitive measures, a significant main effect of group was found for the timing score of the Visual Elevator (VE2) task, $F(2,57)=6.89, p=.002, \eta^{2}=.19$, which assesses individuals' efficiency of attention switching in the visual modality. Post hoc comparisons revealed the [-Per+Pro] participants were significantly slower in switching their attention between task demands than $[+$ Per+Pro $](p=.003)$ and $[+$

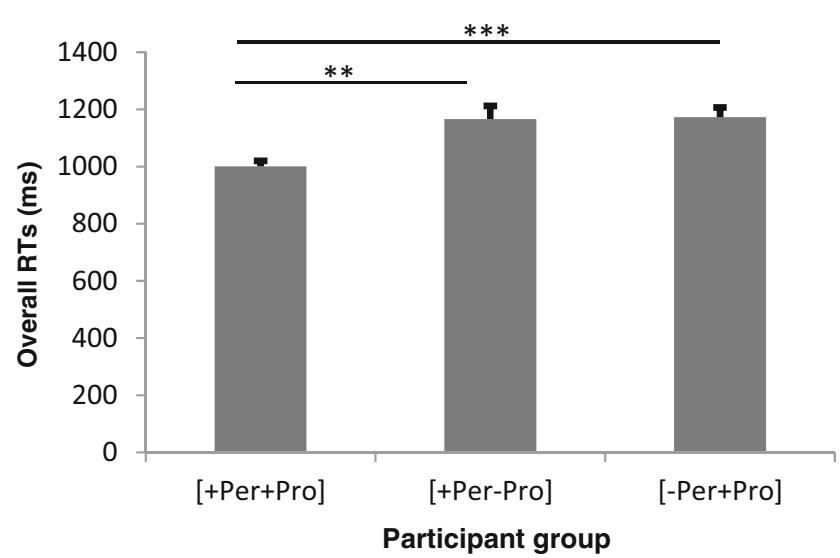

Fig. 2 Mean RTs of tone discrimination. Error bars show \pm 1 standard error. ${ }^{*} p<.05 .{ }^{* *} p<.01 . * * * p<.001$
Per-Pro] ( $p=.020$; see Fig. 3). None of the other measures reliably differed among groups.

Table 3. reveals that the composite scores of TEA visual, TEA auditory, and visual WM were significantly correlated with the overall RT of tone perception, with shorter RT being related to better performance on these cognitive measures. The results also showed that Visual WM correlated more strongly with the overall RT than TEA auditory and TEA Visual, and that the three composite scores were correlated with each other.

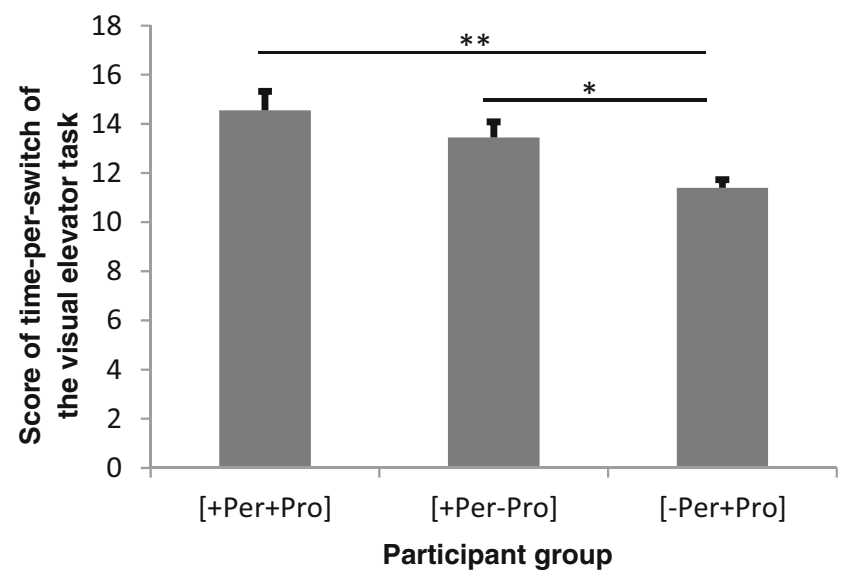

Fig. 3 Mean scores on time-per-switch of the visual elevator task in TEA. Error bars show \pm 1 standard error. ${ }^{*} p<.05 . * * p<.01 . * * * p<.001$ 
Table 3 Correlation among discrimination RTs and cognitive measures

Overall RTs Executive control Auditory stream segregation TEA visual TEA auditory Digit span backward

\begin{tabular}{lllllll}
\hline Executive control & -.207 & & & & & \\
Auditory stream segregation & .035 & -.048 & & & & \\
TEA Visual & $-.380^{* *}$ & -.010 & .168 & $.382^{* *}$ & \\
TEA Auditory & $-.408^{* *}$ & -.095 & .026 & .031 & .196 & $.0311^{*}$ \\
Digit Span Backward & -.078 & .129 & -.007 & .117 & $.486^{* *}$ & .3168 \\
Visual WM & $-.499^{* *}$ & .010 & .17 & \\
\hline
\end{tabular}

${ }^{*} p<.05 .{ }^{* *} p<.01 . * * * p<.001$

The relative contribution of the composite scores of the three main cognitive domains (TEA visual, TEA auditory, and visual WM) to the overall RT was first examined. Results of a stepwise multiple regression confirmed that visual WM and TEA auditory were significant predictors (see Table 4), accounting for $33.2 \%$ of the variance, $F(2,57)=15.69, p<.001$. TEA visual became insignificant as a predictor probably because of its correlations with both visual WM and TEA auditory, and higher correlation between RT and visual WM than TEA visual. Subtest scores comprising visual WM and TEA auditory, respectively, were then entered in a second stepwise regression analysis to delineate which cognitive component(s) contributed to discrimination latency. Individual scores of the visual WM-SS, CD and CA, and TEA auditory-ECD, ECR, and TSC were entered as predictor variables. Only CD, ECR, and CA significantly predicted the discrimination RT, accounting for $31.9 \%, F(2,57)=10.21, p<.001$, of the variance (see Table 5). The CD and CA tasks can be taken to indicate mental processing speed and visual working memory, while ECR indexes attentional switching in the auditory modality. The higher the scores on these three measures, the faster a participant could distinguish two tones.

Table 4 Stepwise regression with Visual WM, TEA Visual and Auditory as predictors and RT as predicted variable

\begin{tabular}{llllll}
\hline Predictor & $\mathrm{B}$ & $S E \mathrm{~B}$ & $\beta$ & $t$ & $p$ \\
\hline Step1 & & & & & \\
$\quad$ Constant & $1,113.07$ & 19.45 & & & \\
$\quad$ Visual WM & -117.98 & 26.59 & -.503 & -4.44 & .000 \\
Step 2 & & & & & \\
$\quad$ Constant & $1,113.07$ & 18.24 & & & \\
$\quad$ Visual WM & -100.73 & 25.59 & -.430 & -3.94 & .000 \\
$\quad$ TEA Auditory & -72.89 & 24.31 & -.327 & -2.99 & .004 \\
\hline
\end{tabular}

Note. $R^{2}=.240$ for Step $1(p<.001) ; \Delta R^{2}=.092$ for Step $2(p<.001)$.

\section{Discussion}

The overall findings have revealed that typically developed native speakers with different patterns of tone perception and production may differ in their ability of visual attention switching, and distinguish speech sounds at different speeds, even for sounds they discriminate with high accuracy. Furthermore, discrimination latencies are related to visual WM (as reflected by the CD and CA tasks), inhibitory control (CA), and auditory attentional switching (VE2 and ECR). These observations suggest that attention and working memory mediate speech processing in a domain-general nature.

The longer discrimination latency demonstrated by $[+$ PerPro] and [-Per+Pro] compared with [+Per+Pro] participants is convergent with the results of Mok et al. (2013). In that study, Cantonese speakers showing a reduced tone space in production were slower in all conditions of a tone discrimination task. The present findings suggest that the differences observed in speech processing might be attributed to individuals' abilities to maintain information temporarily for further operation $(\mathrm{CD}$

Table 5 Stepwise regression with individual test scores in Visual WM and TEA Auditory as predictors and RT as predicted variable

\begin{tabular}{lrrrrl}
\hline Predictor & \multicolumn{1}{l}{$\mathrm{B}$} & \multicolumn{1}{l}{$S E \mathrm{~B}$} & $\beta$ & \multicolumn{1}{l}{$t$} & $p$ \\
\hline Step 1 & & & & & \\
$\quad$ Constant & $1,499.71$ & 110.94 & & & \\
CD & -24.19 & 6.82 & -.422 & -3.55 & .001 \\
Step 2 & & & & & \\
Constant & $1,659.99$ & 121.43 & & & \\
CD & -20.67 & 6.62 & -.361 & -3.12 & .003 \\
ECR & -22.64 & 8.49 & -.308 & -2.67 & .010 \\
Step 3 & & & & & \\
Constant & $1,832.11$ & 131.69 & & & \\
CD & -15.18 & 6.61 & -.265 & -2.29 & .025 \\
ECR & -26.40 & 8.18 & -.359 & -3.23 & .002 \\
CA & -18.10 & 6.70 & -.307 & -2.70 & .009 \\
\hline
\end{tabular}

Note. $\mathrm{CD}=$ Coding; ECR $=$ Elevator Counting with Reversal; $\mathrm{CA}=$ Cancellation. $R^{2}=.164$ for Step $1(p<.01) ; \Delta R^{2}=.080$ for Step 2 $(p<.001) ; \Delta R^{2}=.075$ for Step $3(p<.001)$ 
and CA), to switch attentional focus flexibly (VE2 and ECR), and to inhibit distracting information (CA).

Working memory and mental processing speed reflected by the Coding task accounted for an important proportion of variance in tone discrimination latency. A few studies have reported the contribution of working memory to speech processing (Jacquemot \& Scott, 2006). During a speech perception task, it is expected that listeners need to first decode the auditory signal and transform this time-varying input into an accurate phonemic representation (Cutler \& Clifton, 1999), and maintain the representation in an active state such that operations demanded by the task may be carried out. Our results show that the efficiency in distinctive speech perception seems to correspond to working memory capacity. However, we are aware that the null finding of significant contribution of verbal working memory as measured by digit span backward to speech processing apparently diverged from some previous reports (George \& Coch, 2011; Strait, Kraus, Parbery-Clark, \& Ashley, 2010). In Strait et al. (2010), verbal working memory also indexed by digit span backward was found to be associated with individuals' frequency thresholds in discrimination of nonlinguistic tones. We suggest that digit span tasks, lacking a speeded component, may be less sensitive in capturing the relationship between one's efficiency of allocating memory resources and processing latency.

In addition to working memory, the ability of attentional switching as assessed by the VE2 and ECR tasks in TEA is also shown to be relevant to speech processing speed. Efficient phonological decoding requires accurate mental representations (Ziegler \& Goswami, 2005). As mentioned above, the quality of phonological representations has been proposed to be affected by the flexibility of the attentional system in studies of developmental dyslexia (Lallier et al., 2010). According to this hypothesis, when individuals with relatively sluggish attentional switch have to process sequences of rapidly presented stimuli, their automatic attentional system cannot disengage fast enough from one item to the next, leading to confusion of consecutive sounds, and thereby hindering adequate processing of salient phonological features in speech, resulting in unstable and variant phonemic representations in the mental lexicon. Moreover, the Cancellation task, arguably involving inhibitory control, was also a significant predictor of discrimination latency. Inhibitory control is suggested to play a role in the attunement of speech perception during language development (Kuhl et al., 2008). The ability to ignore acoustic variations in speech that are irrelevant to native language while focusing on language-relevant information is linked to the degree of refinement of phonological representations (Jusczyk, 2002). In the context of speech discrimination, lower quality or less distinctive representations may take longer to be accessed or recognized by incoming acoustic information. This hypothesis predicts an association between faster discrimination of speech sounds with higher attentional switching and/or executive control abilities, which is confirmed by the present findings. It is important to emphasize that the proposal applies to typically-developed speakers, including our [+Per+Pro], [+Per-Pro] and [-Per+Pro] participants, who may vary in the distinctiveness of phonological representations with subtle consequences.

Given the hypothesis just put forth, one may be quick to point out the absence of a relationship between discrimination latency and the measures of auditory stream segregation as well as executive control in the ANT. We suggest that the specific task we employed to evaluate auditory attentional shifting might be more sensitive to detecting deficits in young participants with developmental disorders (see Lallier et al., 2010). Additionally, one may argue that the Cancellation task, which requires individuals to maintain the targets in memory and compare them with each visual stimulus while inhibiting responses to distractors, is more demanding (and hence more sensitive) than the flanker task in the ANT. In the latter, participants could quickly develop a strategy to fixate their attention on the target that always appears in the center of a visual array.

In conclusion, our results have suggested a relationship between individual differences in speech processing and domain-general cognitive abilities of working memory and attentional switch/control among typically developed speakers. The findings have advanced our knowledge from previous works focusing on cognitive processing style ( $\mathrm{Yu}$, 2010) or production strategies (Beddor, 2009). We recognize that this study has focused on the sound change phenomenon of neutralization, and the extent to which individual variations in cognitive functions may account for sound changes in other forms, such as dissimilation and metathesis, would require further investigation.

Acknowledgments This research was supported by a Small Project Fund at the University of Hong Kong (Project titled "Neural correlates and cognitive capability associated with individual variations in tone perception and production in Cantonese-An event-related potential [ERP] study"). S.-P. Law and R. Fung developed the study concept. S.P. Law and J. Ou contributed to the study design. J. Ou carried out data collection, data analysis, and interpretation under the supervision of S.-P. Law. J. Ou drafted the manuscript and S.-P. Law provided the critical revisions. All authors approved the final version of the manuscript for submission.

\section{References}

Andrews, S. (2012). Individual differences in skilled visual word recognition and reading: The role of lexical quality. In J. Adelman (Ed.), Visual word recognition (pp. 151-172). Hove, UK: Psychology Press.

Baker, A., Archangeli, D., \& Mielke, J. (2011). Variability in American English s-retraction suggests a solution to the actuation problem. Language Variation and Change, 23(03), 347-374. 
Bauer, R. S., Cheung, K.-H., \& Cheung, P.-M. (2003). Variation and merger of the rising tones in Hong Kong Cantonese. Language Variation and Change, 15(2), 211-225.

Beddor, P. S. (2009). A coarticulatory path to sound change. Language, $85(4), 785-821$.

Conboy, B. T., Sommerville, J. A., \& Kuhl, P. K. (2008). Cognitive [+ Per+Pro] factors in speech perception at 11 months. Developmental Psychology, 44(5), 1505.

Cutler, A., \& Clifton, C. (1999). Comprehending spoken language: A blueprint of the listener. In C. Brown \& P. Hagoort (Eds.), The neurocognition of language (pp. 123-166). New York, NY: Oxford University Press.

Díaz, B., Baus, C., Escera, C., Costa, A., \& Sebastián-Gallés, N. (2008). Brain potentials to native phoneme discrimination reveal the origin of individual differences in learning the sounds of a second language. Proceedings of the National Academy of Sciences, 105(42), 16083-16088.

Facoetti, A., Trussardi, A. N., Ruffino, M., Lorusso, M. L., Cattaneo, C., Galli, R., . . \& \& Zorzi, M. (2010). Multisensory spatial attention deficits are predictive of phonological decoding skills in developmental dyslexia. Journal of Cognitive Neuroscience, 22(5), 10111025.

Fan, J., McCandliss, B. D., Sommer, T., Raz, A., \& Posner, M. (2002). Testing the efficiency and independence of attentional networks. Journal of Cognitive Neuroscience, 14, 340-347.

George, E. M., \& Coch, D. (2011). Music training and working memory: An ERP study. Neuropsychologia, 49(5), 1083-1094.

Haesen, B., Boets, B., \& Wagemans, J. (2011). A review of behavioural and electrophysiological studies on auditory processing and speech perception in autism spectrum disorders. Research in Autism Spectrum Disorders, 5(2), 701-714.

Hari, R., \& Renvall, H. (2001). Impaired processing of rapid stimulus sequences in dyslexia. Trends in Cognitive Sciences, 5(12), 525532 .

Holt, L. L., \& Lotto, A. J. (2010). Speech perception as categorization. Attention, Perception, \& Psychophysics, 72(5), 1218-1227.

Jacquemot, C., \& Scott, S. K. (2006). What is the relationship between phonological short-term memory and speech processing? Trends in Cognitive Sciences, 10(11), 480-486.

Jusczyk, P. W. (2002). How infants adapt speech-processing capacities to native-language structure. Current Directions in Psychological Science, 11(1), 15-18.

Koenig, L. L., Lucero, J. C., \& Perlman, E. (2008). Speech production variability in fricatives of children and adults: Results of functional data analysis. The Journal of the Acoustical Society of America, 124(5), 3158-3170.

Kuhl, P. K., Conboy, B. T., Coffey-Corina, S., Padden, D., RiveraGaxiola, M., \& Nelson, T. (2008). Phonetic learning as a pathway to language: new data and native language magnet theory expanded (NLM-e). Philosophical Transactions of the Royal Society, B: Biological Sciences, 363(1493), 979-1000.
Labov, W. (1994). Principles of linguistic change. Vol. 1: Internal factors. Oxford, UK: Blackwell.

Lallier, M., Tainturier, M. J., Dering, B., Donnadieu, S., Valdois, S., \& Thierry, G. (2010). Behavioral and ERP evidence for amodal sluggish attentional shifting in developmental dyslexia. Neuropsychologia, 48(14), 4125-4135.

Law, S. P., Fung, R., \& Kung, C. (2013). An ERP study of good production vis-à-vis poor perception of tones in Cantonese: Implications for top-down speech processing. PloS One, 8(1), e54396.

Lengeris, A., \& Hazan, V. (2010). The effect of native vowel processing ability and frequency discrimination acuity on the phonetic training of English vowels for native speakers of Greek. The Journal of the Acoustical Society of America, 128(6), 3757-3768.

Lev-Ari, S., \& Peperkamp, S. (2014). The influence of inhibitory skill on phonological representations in production and perception. Journal of Phonetics, 47, 36-46.

Mok, P. P., Zuo, D., \& Wong, P. W. (2013). Production and perception of a sound change in progress: Tone merging in Hong Kong Cantonese. Language Variation and Change, 25(03), 341-370.

Näätänen, R., Paavilainen, P., Rinne, T., \& Alho, K. (2007). The mismatch negativity (MMN) in basic research of central auditory processing: a review. Clinical Neurophysiology, 118(12), 2544-2590.

Parbery-Clark, A., Skoe, E., Lam, C., \& Kraus, N. (2009). Musician enhancement for speech-in-noise. Ear and Hearing, 30(6), 653661.

Polich, J. (2003). Overview of P3a and P3b. In J. Polich (Ed.), Detection of change: Event-related potential and fMRI findings (pp. 83-98). Boston, MA: Kluwer.

Robertson, I. H., Ward, T., Ridgeway, V., \& Nimmo-Smith, I. (1994). The test of everyday attention: TEA. Bury St. Edmunds, UK: Thames Valley Test Company.

Ruffino, M., Trussardi, A. N., Gori, S., Finzi, A., Giovagnoli, S., Menghini, D., . . \& \& Facoetti, A. (2010). Attentional engagement deficits in dyslexic children. Neuropsychologia, 48(13), 3793-3801.

Strait, D. L., Kraus, N., Parbery-Clark, A., \& Ashley, R. (2010). Musical experience shapes top-down auditory mechanisms: Evidence from masking and auditory attention performance. Hearing Research, 261(1), 22-29.

Veldre, A., \& Andrews, S. (2014). Lexical quality and eye movements: Individual differences in the perceptual span of skilled adult readers. The Quarterly Journal of Experimental Psychology, 67(4), 703727.

Wechsler, D. (2010). Wechsler Adult Intelligence Scale (4th ed.). San Antonio, TX: Pearson/PsychCorp.

Yip, M. (2002). Tone. Cambridge, UK: Cambridge University Press.

Yu, A. C. L. (2010). Perceptual compensation is correlated with individuals' "autistic" traits: Implications for models of sound change. PLOS ONE, 5(8), e11950.

Ziegler, J. C., \& Goswami, U. (2005). Reading acquisition, developmental dyslexia, and skilled reading across languages: A psycholinguistic grain size theory. Psychological Bulletin, 131(1), 3. 